\title{
Transformation operators for the Schrödinger equation with a linearly increasing potential
}

\author{
Khatira E. Abbasova · Agil Kh. Khanmamedov
}

Received: 08.08.2019 / Revised: 10.01.2020 / Accepted: 03.02.2020

\begin{abstract}
The Schrödinger equation with a linearly growing potential is considered. Using transformation operators, we obtain representations of solutions of this equation with conditions at infinity. Estimates for the kernels of the transformation operators are obtained.
\end{abstract}

Keywords. Schrödinger equation - transformation operator · Airy functions - Stark equation.

Mathematics Subject Classification (2010): 34A55, 34B20, 34L05.

\section{Introduction}

In many aspects of the theory of inverse problems of spectral analysis, an important role is played by so-called transformation operators (see [8], [10] and the references therein). These operators arose from the general ideas of the theory of generalized shift operators created by Delsarte [3]. For arbitrary SturmLiouville equations, transformation operators were constructed by Povzner [10]. Marchenko [8] used transformation operators for studying inverse spectral problems and the asymptotic behavior of the spectral function of the singular SturmLiouville operator. Levin [7] introduced transformation operators of a new form that preserve the asymptotic expansions of solutions at infinity. Marchenko [8] used them to solve the inverse problem of scattering theory. Similar problems for the Schrödinger equation with unbounded potentials were considered in [2], [4], [9], [12].

We consider the differential equation

$$
-y^{\prime \prime}+|x| y+q(x) y=\lambda y,-\infty<x<\infty, \lambda \in C .
$$

where the real potential $q(x)$ satisfies the conditions

$$
q(x) \in C(-\infty,+\infty), \quad \int_{-\infty}^{\infty}|x q(x)| d x<\infty
$$

In the present paper, using transformation operators, we obtain representations of solutions of this equation with conditions at infinity. The results obtained can be used to solve

Kh.E. Abbasova

Azerbaijan State University of Economics (UNEC), AZ 1001, Baku, Azerbaijan

E-mail: abbasova_xatira@unec.edu.az

A.Kh. Khanmamedov

Institute of Mathematics and Mechanics of NAS of Azerbaijan, AZ 1141, Baku, Azerbaijan

E-mail: agil_khanmamedov@yahoo.com 
inverse spectral problems for an equation (1.1). Note that for $x \geq 0$, equation (1.1) turns into the one-dimensional Stark equation. Some questions of the spectral theory of the onedimensional Stark equation were studied in [5], [6], [9].

\section{The transformation operators}

In what follows, we deal with special functions satisfying the Airy equation

$$
-y^{\prime \prime}+z y=0 .
$$

It is well known (e.g., see [12]) that this equation has two linearly independent solutions $A i(z)$ and $B i(z)$ with the initial conditions

$$
\begin{gathered}
A i(0)=\frac{1}{3^{\frac{2}{3}} \Gamma\left(\frac{2}{3}\right)}, A i^{\prime}(0)=\frac{1}{3^{\frac{1}{3}} \Gamma\left(\frac{1}{3}\right)}, \\
B i(0)=\frac{1}{3^{\frac{1}{6}} \Gamma\left(\frac{2}{3}\right)}, B i^{\prime}(0)=\frac{3^{\frac{1}{6}}}{\Gamma\left(\frac{1}{3}\right)} .
\end{gathered}
$$

The Wronskian $\{A i(z), B i(z)\}$ of these functions satisfies

$$
\{A i(z), B i(z)\}=A i(z) B i^{\prime}(z)-A i^{\prime}(z) B i(z)=\pi^{-1} .
$$

Both functions are entire functions of order $\frac{3}{2}$ and type $\frac{2}{3}$. We have (see [1]) asymptotic equalities for $|z| \rightarrow \infty$

$$
\begin{gathered}
A i(z) \sim \pi^{-\frac{1}{2}} z^{-\frac{1}{4}} e^{-\zeta}\left[1+O\left(\zeta^{-1}\right)\right],|\arg z|<\pi, \\
A i(-z) \sim \pi^{-\frac{1}{2}} z^{-\frac{1}{4}} \sin \left(\zeta+\frac{\pi}{4}\right)\left[1+O\left(\zeta^{-1}\right)\right],|\arg z|<\frac{2 \pi}{3}, \\
B i(z) \sim \pi^{-\frac{1}{2}} z^{-\frac{1}{4}} e^{\zeta}\left[1+O\left(\zeta^{-1}\right)\right],|\arg z|<\frac{\pi}{3}, \\
B i(-z) \sim \pi^{-\frac{1}{2}} z^{-\frac{1}{4}} \cos \left(\zeta+\frac{\pi}{4}\right)\left[1+O\left(\zeta^{-1}\right)\right],|\arg z|<\frac{2 \pi}{3} .
\end{gathered}
$$

where $\zeta=\frac{2}{3} z^{\frac{3}{2}}$. In what follows we will need special solutions of the unperturbed equation

$$
-y^{\prime \prime}+|x| y=\lambda y,-\infty<x<\infty, \lambda \in C .
$$

Lemma 2.1 For any $\lambda$ from the complex plane, equation (2.1) has solutions $\psi_{ \pm}(x, \lambda)$ in the form

$$
\begin{aligned}
& \psi_{+}(x, \lambda)=\left\{\begin{array}{c}
A i(x-\lambda), x \geq 0, \\
-\pi(A i(-\lambda) B i(-\lambda))^{\prime} A i(-x-\lambda)- \\
2 \pi A i(-\lambda) A i^{\prime}(-\lambda) B i(-x-\lambda), x<0,
\end{array}\right. \\
& \psi_{-}(x, \lambda)=\left\{\begin{array}{l}
-\pi(A i(-\lambda) B i(-\lambda))^{\prime} A i(x-\lambda)- \\
-2 \pi A i(-\lambda) A i^{\prime}(-\lambda) B i(x-\lambda), x \geq 0, \\
A i(-x-\lambda), x<0 .
\end{array}\right.
\end{aligned}
$$


Proof. Obviously, when $x \geq 0$ one of the solutions of equation (2.1) is function $A i(x-\lambda)$. On the other hand, for $x \leq 0$ any solution of equation (2.1) can be represented as

$$
\alpha A i(-x-\lambda)+\beta B i(-x-\lambda) .
$$

If we glue these solutions at a point $x=0$, we get

$$
\alpha=-\pi(A i(-\lambda) B i(-\lambda))^{\prime}, \beta=-2 \pi A i(-\lambda) A i^{\prime}(-\lambda) .
$$

Thus, formula (2.2) is established. Formula (2.3) is derived similarly.

The lemma is proved.

We shall use the following notation

$$
\sigma_{ \pm}(x)= \pm \int_{x}^{ \pm \infty}|-t+| t|+q(t)| d t .
$$

In the following theorem the representation of solution from the equation (1.1) is found by means of transformation operator.

Theorem 2.1 If the potential $q(x)$ satisfies conditions (1.2), then for any $\lambda$ from the complex plane equation (1.1) has a solution $f_{+}(x, \lambda)$ that can be represented in the form

$$
f_{+}(x, \lambda)=\psi_{+}(x, \lambda)+\int_{x}^{\infty} K_{+}(x, t) \psi_{+}(t, \lambda) d t
$$

where kernel $K_{+}(x, t)$ is continuous function and satisfies relations

$$
\begin{gathered}
K_{+}(x, t)=O\left(\sigma_{+}\left(\frac{x+t}{2}\right)\right), x+t \rightarrow \infty, \\
K_{+}(x, x)=\frac{1}{2} \int_{x}^{\infty}(|t|-t+q(t)) d t .
\end{gathered}
$$

Proof. We rewrite the perturbed equation (1.1) in the form

$$
-y^{\prime \prime}+x y+Q(x) y=\lambda y,-\infty<x<\infty .
$$

where $Q(x)=|x|-x+q(x)$. Obviously, the $Q(x)$ function for all $x>a, a>-\infty$ satisfies the condition

$$
Q \in C(-\infty,+\infty), \quad \int_{a}^{\infty}|x Q(x)| d x<\infty .
$$

Let $f_{+}(x, \lambda)$ be solution of equation (2.7) with the asymptotic behavior

$$
f_{+}(x, \lambda)=f_{0}(x, \lambda)(1+o(1)), x \rightarrow \infty,
$$

where $f_{0}(x, \lambda)=A i(x-\lambda)$. Subject to the conditions (2.8), such solution exist, is determined uniquely by its asymptotic behavior. With the aid of operator transformations, we have the representation

$$
f_{+}(x, \lambda)=f_{0}(x, \lambda)+\int_{x}^{\infty} K(x, t) f_{0}(t, \lambda) d t .
$$

Moreover, the kernel $K(x, t)$ is a continuous function and satisfies the following relations

$$
K(x, t)=O\left(\sigma_{+}\left(\frac{x+t}{2}\right)\right), x+t \rightarrow \infty,
$$




$$
K(x, x)=\frac{1}{2} \int_{x}^{\infty} Q(t) d t .
$$

In addition, rewriting the unperturbed equation (2.1) in the form

$$
-y^{\prime \prime}+x y+Q_{0}(x) y=\lambda y,-\infty<x<\infty,
$$

where $Q_{0}(x)=|x|-x$, we obtain

$$
\psi_{+}(x, \lambda)=f_{0}(x, \lambda)+\int_{x}^{\infty} K_{0}(x, t) f_{0}(t, \lambda) d t .
$$

Moreover, in this case, $K_{0}(x, t)$ satisfies the identity $K_{0}(x, t) \equiv 0, x \geq 0$. From the well-known properties of the transformation operators it follows that (see [8]) the function $f_{0}(x, \lambda)$ also admits the representation

$$
f_{0}(x, \lambda)=\psi_{+}(x, \lambda)+\int_{x}^{\infty} \widetilde{K}_{0}(x, t) \psi_{+}(t, \lambda) d t,
$$

where the kernels $K_{0}(x, t), \widetilde{K}_{0}(x, t)$ are connected by the equality

$$
K_{0}(x, t)+\widetilde{K}_{0}(x, t)+\int_{x}^{t} \widetilde{K}_{0}(x, u) K_{0}(u, t) d u=0 .
$$

Substituting the expression (2.12) from the $f_{0}(x, \lambda)$ in (2.8), we get

$$
\begin{aligned}
& f_{+}(x, \lambda)=\psi_{+}(x, \lambda)+\int_{x}^{\infty} K(x, t)\left[\psi_{+}(t, \lambda)+\int_{t}^{\infty} \widetilde{K}_{0}(t, u) \psi_{+}(u, \lambda) d u\right] d t= \\
& =\psi_{+}(x, \lambda)+\int_{x}^{\infty} K(x, t) \psi_{+}(t, \lambda) d t+\int_{x}^{\infty} K(x, t) \int_{t}^{\infty} \widetilde{K}_{0}(t, u) \psi_{+}(u, \lambda) d u d t= \\
& =\psi_{+}(x, \lambda)+\int_{x}^{\infty} K(x, t) \psi_{+}(t, \lambda) d t+\int_{x}^{\infty}\left(\int_{x}^{t} K(x, u) \widetilde{K}_{0}(u, t) d u\right) \psi_{+}(t, \lambda) d t .
\end{aligned}
$$

Setting

$$
K_{+}(x, t)=K(x, t)+\int_{x}^{t} K(x, u) \widetilde{K}_{0}(u, t) d u,
$$

one can recast the last relation in the form

$$
f_{+}(x, \lambda)=\psi_{+}(x, \lambda)+\int_{x}^{\infty} K_{+}(x, t) \psi_{+}(t, \lambda) d t .
$$

Formula (2.5) is a straightforward consequence of (2.10), (2.13), (2.14). Taking $t=x$ in the equality (2.14), we find that $K_{+}(x, t)=K(x, t)$. Whence, by virtue of (2.11), formula (2.6) follows.

The theorem is proved.

The following theorem is proved in a similar way.

Theorem 2.2 If the potential $q(x)$ satisfies condition (1.1), then, for all values of $\lambda$, equation (1.2) has a solution $f_{-}(x, \lambda)$ representable as

$$
f_{-}(x, \lambda)=\psi_{-}(x, \lambda)+\int_{-\infty}^{x} K_{-}(x, t) \psi_{-}(t, \lambda) d t,
$$

where the kernel $K_{-}(x, t)$ is continuous function and satisfy the following conditions

$$
\begin{aligned}
& K_{-}(x, t)=O\left(\sigma_{-}\left(\frac{x+t}{2}\right)\right), x+t \rightarrow \infty \\
& K_{-}(x, x)=\frac{1}{2} \int_{-\infty}^{x}(|t|+t+q(t)) d t .
\end{aligned}
$$




\section{References}

1. Abramowitz, M., Stegun, I.: Handbook of Mathematical Functions with Formulas, Graphs, and Mathematical Tables, Dover Publications, New York, (1972).

2. Bagirova, S.M., Khanmamedov, A.Kh.: The inverse spectral problem for the perturbed harmonic oscillator on the entire axis, Proc. Inst. Math. Mech. Natl. Acad. Sci. Azerb. 44(2), 285-294 (2018).

3. Delsarte, J.: Sur une extension de la formule de Taylor, J. Math. Pures Appl. 17, 213-231 (1938).

4. Gasymov, M.G., Mustafaev B.A.: Inverse scattering problem for an anharmonic equation on the semiaxis, Dokl. Akad. Nauk SSSR 228(1), 11-14 (1976).

5. Korotyaev, E.L.: Resonances for 1D Stark operators, J. Spectr. Theory 7(3), 633-658 (2017).

6. Korotyaev, E.L.: Asymptotics of resonances for 1D Stark operators, Lett. Math. Phys. 118(5), 1307-1322 (2018).

7. Levin, B.Ya. : Transformation of Fourier and Laplace type by means of solutions of a differential equation of second order, Dokl. Akad. Nauk SSSR 106(2), 187-190 (1956).

8. Marchenko, V.A.: Sturm-Liouville operators and their applications, Naukova Dumka, Kiev, (1977) [in Russian].

9. Masmaliev, G.M., Khanmamedov A.Kh.: Transformation operators for perturbed harmonic oscillators, Math. Notes 105(5), 728-733 (2019).

10. Povzner, A.Ya.: On differential equations of Sturm-Liouville type on a half-axis, (Russian) Mat. Sbornik N.S. 23(65), 3-52 (1948).

11. Savchuk, A.M., Shkalikov A.A.: Spectral properties of the complex Airy operator on the half-line, Funct. Anal. Appl. 51(1), 66-79 (2017).

12. Yishen, Li.: One special inverse problem of the second-order differential equation for the whole real axis, Chinese Ann. Math. 2(2), 147-155 (1981). 